\title{
Role of CRP in the Evaluation and Diagnostic use in Assessment of Neonatal Sepsis in Tertery care Hospital
}

\author{
Badri Kumar Gupta ${ }^{1 *}$, Nand Kishor Gupta ${ }^{2}$
}

${ }^{1}$ Professor, Department of Paediatrics, Universal College of Medical Sciences, Bhairahawa, Rupandehi, Nepal

${ }^{2}$ Department of Anatomy, Uttarpradesh Universal College of Medical Sciences, Saifai, Etawah, Uttar Pradesh, India

DOI: $10.36347 /$ sjams.2020.v08i05.040

| Received: 12.04 .2020 | Accepted: 20.04.2020 | Published: 30.05 .2020

*Corresponding author: Badri Kumar Gupta

Abstract

Original Research Article

Introduction: Neonatal sepsis, a clinical syndrome of bacteremia with systemic signs and symptoms of infection in the first 4 weeks of life is a major cause of morbidity and mortality in newborn. Early diagnosis is critical, as sepsis can progress more rapidly in neonates than in adults. In a developing country like Nepal, where proper medical care and obstetrical facilities are still scarce in the rural areas. With limited resources, early diagnosis and treatment if crucial, may not be achieved. C-reactive protein (CRP) is the most common diagnostic marker of infection. The aims of this study is to evaluate the role of CRP in the diagnosis of neonatal sepsis. Material and Methods: This prospective observational study were done in the department of paediatrics, UCMS-TH, Bhairahawa from May 2019 to March 2020. Clinically suspected neonatal sepsis cases were enrolled in the study. Venous blood were collected before giving any intravenous fluid or antibiotics for complete blood counts, CRP levels, blood sugar and blood culture and send to laboratory within half hour of collection. All patients included in this study were treated accordingly and followed up strictly. Results: A total of 440 patients clinically diagnosed as neonatal sepsis were studied. The mean age was found $10.1 \pm 8.5$ days with range from 1 to 28 days and more than half $(51.6 \%)$ babies belonged to age $\leq 7$ days. More than two third $71.4 \%$ babies were male and $28.6 \%$ were female. $236(53.6 \%)$ babies were found CRP positive and 204(46.4\%) were CRP negative. $25.5 \%$ babies were blood culture positive and $74.5 \%$ babies were culture negative.364 (82.73\%) babies were cured and $76(17.27 \%)$ were died. Conclusion: CRP is a simple, relatively cheap and sensitive study in prediction of neonatal sepsis. It can be useful test in settings with limited resources. : Serum CRP is the most sensitive marker of sepsis.

Keywords: C-reactive protein, Antibiotic, Neonate, Sepsis.

Copyright @ 2020: This is an open-access article distributed under the terms of the Creative Commons Attribution license which permits unrestricted use, distribution, and reproduction in any medium for non-commercial use (NonCommercial, or CC-BY-NC) provided the original author and source are credited.

\section{INTRODUCTION}

Sepsis is the commonest cause of neonatal mortality; it is responsible for about $30-50 \%$ of the total neonatal deaths in developing countries [1]. It is estimated that up to $20 \%$ of neonates develop sepsis and approximately $1 \%$ die of sepsis related causes [2]. All neonates suspected to have sepsis should have a septic screen to corroborate the diagnosis. However, the decision to start antibiotics need not be conditional to the sepsis screen result, if there is a strong clinical suspicion of sepsis. The clinical diagnosis of neonatal sepsis is difficult because the signs and symptoms are not always specific. There is no laboratory test with $100 \%$ sensitivity and specificity [3]. Blood culture has been considered the gold standard for confirmation of diagnosis but the results are available only after 48-72 hours. Moreover, in many cases blood culture fails to detect the offending organism/bacteria. So, the search for a reliable test continues, especially one that is useful in culture-negative cases [4]. It encompasses various systemic infections of the newborn such as septicaemia, meningitis, pneumonia, arthritis, osteomyelitis and urinary tract infections [5]. Neonatal mortality rate of Nepalis 33per 1,000 live births and sepsis is one of the leading cause. In B.S year 2070/71, of the total neonates presenting to government health facilities, $13.9 \%$ had possible bacterial infection and $42.1 \%$ had local bacterial infection. The major causes of neonatal deaths in Nepal are infection, birth asphyxia, preterm birth, and hypothermia [6].

Despite advances in maternal and neonatal care, infection remains a frequent and important cause of neonatal and infant mortality and morbidity [7]. Neonatal mortality is associated with about $41 \%$ of all death among under-five children [8]. Newborn infants, especially sick or preterm infants, are at risk of developing severe infections (such as bloodstream 
infections) during their stay on neonatal units. Infections are often difficult to diagnose early with certainty, and quick tests such as measuring the blood level of a protein that responds to infection (called Creactive protein) are sometimes used to help make an earlier diagnosis. Neonatal sepsis evaluation, a common occurrence in the Neonatal Intensive Care Unit (NICU), is usually comprised a complete blood count (CBC), blood culture and possibly cerebrospinal fluid (CSF), and urine cultures. When an infant appears ill the clinical findings such as temperature instability, respiratory distress, apnea, bradycardia, and hypotension are nonspecific [10, 11]. Sometimes, especially if noted in isolation, these findings are transient, and no infection is confirmed. If an organism is isolated from a sterile site such as blood or CSF, the treatment course is straightforward. For the majority of remaining infants, the nonspecific symptoms resolve at some point after sepsis evaluation, but antibiotics have already been started. Often an organism is not isolated from the various specimens collected, bringing uncertainty of when to discontinue antibiotic treatment. The use of biomarkers, such as C-reactive protein (CRP), can assist in sepsis management decision-making [12]. The use of rapid blood testing for CRP has become common in pediatrics to help evaluate infection in children and increasingly more common during the evaluation of sepsis in neonates [13]. CLASSIFICATIONOF NEONATAL SEPSIS: Neonatal sepsis can be classified into two major categories depending up on the onset of symptoms, EARLY ONSET SEPSIS (EOS): It presents within first 72 hours of life. In severe cases, the neonate may be symptomatic at birth. Infants with EOS usually present with respiratory distress and pneumonia. The source of infection is generally the maternal genital tract. LATE ONSET SEPSIS(LOS): It usually presents after72 hours of age. The source of infection in LOS is either nosocomial (hospital acquired) or community acquired and neonates usually present with septicemia, pneumonia or meningitis.

\section{Material ANd Methods}

It is a prospective observational study, conducted during May 2019 to March 2020 in the Department of Paediatrics, UCMS. A detailed history and thorough physical examination were done in each neonate on admission. History included age of newborn, sex, gestational age, $\mathrm{h} / \mathrm{o}$ prolonged rupture of membrane (PROM), intrapartum fever or fever 3 days before delivery, per vaginal foul smelling discharge, prolonged labour and features of sepsis. Physical examination included respiratory rate, heart rate, temperature, chest indrawing, grunting, cyanosis, convulsion, breath sound, added sound, weight, jaundice, bleeding manifestation, status of fontanelles, umbilicus and capillary refill time. Venous blood was collected before giving any intravenous fluid or antibiotics for blood sugar, complete blood counts, CRP levels and blood culture and sensitivity. Sample was send to labouratory with in half hour of collection. CRP levels $>6 \mathrm{mg} / \mathrm{L}$ considered as positive and < $6 \mathrm{mg} / \mathrm{L}$ considered as negative. All patients included in this study were treated accordingly and followed up strictly. The outcome and relevant data from history, physical examination and investigations were recorded in predesigned questionnaire.

\section{Inclusion Criteria}

Neonates were enrolled on the basis of signs and symptoms of clinical sepsis after through clinical examination and proper history taking. The clinical criteria considered were - poor feeding, irritability / excessive cry, lethargy poor cry and reflexes, fever, hypothermia, jaundice, vomiting, abdominal distension, tachypnoea and grunting, convulsions, diarrhea, pustules, cyanosis, bulged fontanalle, bleeding, poor perfusion / shock, apnea. Also significant predisposing factors for presumed early onset sepsis was taken into consideration during inclusion of cases.

\section{Exclusion Criteria}

Neonates who received antibiotics before Admission, Neonates who died before work up was complete, Neonates who underwent surgery, Congenital anomalies e.g. tracheoesophageal fistula, lobar agenesis, malrotation of the gut, complex heart diseases, neural tube defects etc. Inborn errors of metabolism.

\section{Investigations Done - Sepsis Screening}

- Absolute Neutrophil Count (ANC) $:<1800 / \mathrm{cu}$ $\mathrm{mm}$

- Immature to Total Neutrophil (I/T) RATIO :> 0.2(immature neutrophils/ANC), highlysensitive of NNS,

- Total Leukocyte Count (TLC) : 5000/cu mmor $>20,000 / \mathrm{cu} \mathrm{mm}$

- $\quad \mathrm{CRP}:>6 \mathrm{mg} / \mathrm{dL}$

- Micro ESR ( $\mu$-ESR): $>15 \mathrm{~mm} / 1^{\mathrm{st}} \mathrm{hr}$, specificbut moderate sensitivity

- $\quad$ Platelets $<150000 \mathrm{cu} \mathrm{mm}$

Blood Culture

NOTE: $(\geq 2)$ positive screening parameters (TLC, ANC, I/Tratio, CRP, u-ESR), platelets counts taken as sepsis screen positive ( $\mathrm{Sn} 93 \%$, PPV39\%,>2parameters-NPV99\%) and that neonate is with sepsis.

\section{Ethical Clearance}

The approval of Institutional Review Committee of Universal College of Medical Sciences, Bhairahawa, Nepal was taken before the initiation of experiment. Registration No. UCMS/IRC/117/19. All the protocols and experiments were conducted in compliance with the ethical principles and guidelines.

\section{Statistical Analysis}

Data were processed manually and analyzed with the help of SPSS (Statistical package for social 
sciences) Version 16.0. Quantitative data were expressed as mean and standard deviation. Qualitative data were expressed as frequency and percentage and comparison carried by Chi-square $(\chi 2)$ test. A probability (p) value of $<0.05$ was considered statistically significant and $\mathrm{p}<0.01$ was considered highly significant but $\mathrm{p}>0.05$ taken as non-significant.

\section{RESULTS}

A total of 440 patients clinically diagnosed as neonatal sepsis were studied. The mean age was found $10.1 \pm 8.5$ days with range from 1 to 28 days and more than half $(51.6 \%)$ babies belonged to age $\leq 7$ days. More than two third $(71.4 \%)$ babies were male and $28.6 \%$ were female. $236(53.6 \%)$ babies were found CRP positive and 204(46.4\%) were negative CRP. $25.5 \%$ babies were blood culture positive and $74.5 \%$ babies were culture negative.

Table-1: Demographic data of neonatal sepsis: $(n=440)$

\begin{tabular}{|l|l|l|l|l|}
\hline S. N. & Category & Distribution & Frequency & Percentage \\
\hline 1 & Religion & Hindu & 282 & $64.1 \%$ \\
& & Muslim & 110 & $25.0 \%$ \\
& & Others & 48 & $10.9 \%$ \\
\hline 2 & Sex & Male & 314 & $71.4 \%$ \\
& & Female & 126 & $28.6 \%$ \\
\hline 3 & Mode of delivery & NVD & 232 & $52.7 \%$ \\
& & LSCS & 208 & $47.3 \%$ \\
\hline 4 & Maternal risk factor for sepsis & Yes & 112 & $30.0 \%$ \\
& & No & 308 & $70.0 \%$ \\
\hline 5 & PV leakage >18hours & Yes & 52 & $11.8 \%$ \\
& & No & 388 & $88.2 \%$ \\
\hline 6 & Maturity of baby & Pre-term & 82 & $18.6 \%$ \\
& & Term & 342 & $77.7 \%$ \\
& & Post-term & 16 & $3.6 \%$ \\
\hline 7 & Weight for Age & SGA & 68 & $15.5 \%$ \\
& & AGA & 366 & $83.2 \%$ \\
& & LGA & 6 & $1.4 \%$ \\
\hline 8 & Low birth weight & YES & 82 & $18.6 \%$ \\
& & NO & 358 & $81.4 \%$ \\
\hline
\end{tabular}

Table 1 showed $282 \quad(64.1 \%)$ Hindu, $110(25.0 \%)$ Muslim, and others were 48(10.9\%). $314(71.4 \%)$ male and $126(28.6 \%)$ female neonate with male to female ratio of $2.5: 1$. However 232(52.7\%) were delivered by normal vaginal delivery and $208(47.3 \%)$ were delivered by caesarian section. maternal risk factor for sepsis were $132(30.0 \%)$ and without risk factor for sepsis $308(70.0 \%)$. PV leakage were present in $52(11.8 \%)$ cases. $82(18.6 \%)$ neonate were born low birth weight and $358(81.4 \%$ ) were normal weight $(>2.5 \mathrm{~kg}) .82(18.6 \%)$ were preterm, $342(77.7 \%)$ were term baby and $16(3.6 \%)$ cases were post-term baby.

Table-2: Common clinical features in suspected neonatal sepsis

\begin{tabular}{|l|l|l|}
\hline & No. & Percentage \\
\hline Respiratory distress & $\mathbf{9 8}$ & $\mathbf{2 2 . 2 7}$ \\
\hline Refuse feeding & $\mathbf{8 7}$ & $\mathbf{1 9 . 7 7}$ \\
\hline Lethargic & $\mathbf{6 4}$ & $\mathbf{1 4 . 5 4}$ \\
\hline Fever & $\mathbf{5 6}$ & $\mathbf{1 2 . 7 2}$ \\
\hline Hypothermia & $\mathbf{2 8}$ & $\mathbf{6 . 3 6}$ \\
\hline Vomiting & $\mathbf{5 6}$ & $\mathbf{1 2 . 7 2}$ \\
\hline Seizure & $\mathbf{2 3}$ & $\mathbf{5 . 2 2}$ \\
\hline Sclerma & $\mathbf{1 2}$ & $\mathbf{2 . 7 2}$ \\
\hline Abdominal distension & $\mathbf{1 6}$ & $\mathbf{3 . 6}$ \\
\hline Total & $\mathbf{4 4 0}$ & $\mathbf{1 0 0 \%}$ \\
\hline
\end{tabular}

Among the patients with suspected neonatal sepsis, the most common presenting clinical feature was respiratory distress followed by feeding problems and lethargic.

Table-3: CRP valve in suspected neonatal sepsis

\begin{tabular}{|l|l|l|}
\hline CRP & No. of patients & No (\%) \\
\hline Positive & 236 & $53.6 \%$ \\
\hline Negative & 204 & $46.4 \%$ \\
\hline Total & 440 & $100 \%$ \\
\hline
\end{tabular}

Out of total 440 suspected neonatal sepsis, 236(53.6\%) were CRP positive and 204(46.4\%) were CRP negative.

Table-4: Distribution of isolated organism

\begin{tabular}{|l|l|l|}
\hline Organisms & Number & Percentage \\
\hline Coagulase negative & 44 & $43.13 \%$ \\
\hline Klebsiella & 24 & $23.52 \%$ \\
\hline Actinobacter species & 12 & $11.76 \%$ \\
\hline Staphylocus epidermis & 4 & $3.92 \%$ \\
\hline Entercoccus species & 4 & $3.92 \%$ \\
\hline MR staphylococci species & 12 & $11.76 \%$ \\
\hline Grampositivecoccus CONS & 2 & $1.96 \%$ \\
\hline Total & $\mathbf{1 0 2}$ & $\mathbf{1 0 0 . 0 \%}$ \\
\hline
\end{tabular}


Of the 440 cases were evaluated for sepsis, the blood culture was positive in $102(24.9 \%)$ cases. In which Coagulasenegative Staphylococci 44(43.3\%) and Kleibsella species 24(23.52\%) were the most common Gram positive and Gram negative organisms.

Table-5: Relationship between CRP and glucose level

\begin{tabular}{|c|c|c|c|c|}
\hline CRP & $<45$ & $\mathbf{4 5 - 1 4 5}$ & $\mathbf{> 1 4 5}$ & Total \\
\hline Positive & 80 & 122 & 34 & 236 \\
\hline Negative & 76 & 124 & 4 & 204 \\
\hline Total & 156 & 246 & 38 & 440 \\
\hline
\end{tabular}

Majority $(51.7 \%)$ of CRP positive patients were found normoglycemic, $33.9 \%$ were found hypoglycemic and only $14.4 \%$ were found hyperglycemic.

\section{Discussion}

Out of 440 suspected neonatal sepsis patients, $314(71.4 \%)$ were males and $126(28,6 \%)$ were females. Among the patients with suspected neonatal sepsis, the most common presenting clinical feature was respiratory distress followed by feeding problems and lethargic. In this study, Coagulase negative staphylococcus (CONS) (43.13\%), Klebsiella species (23.52\%), Acinitobacter species (11.76\%), Pseudomonas aeruginosa (3.92\%), Enterococcus species $(3.2 \%)$ and Methacillin resistant Staphylococcus aureus $(11.76 \%)$ were the common organisms associated with sepsis. In a similar study done in India most prevalent organisms were coagulase negative staphylococcus (CONS) followed by Coagulase positive staphylococcus, streptococcus fecalis, alpha- hemolytic Streptococcus, klebsiella, proteus, E.coli and Candida albicans [14]. The causative organisms in neonatal sepsis vary from place to place and the frequency of the causative organisms is different in different hospitals and even in the same hospital at different time. The other published data in Nepal on the subject shows E.coli as the most common isolate $[15,16]$.

Definitive diagnosis rests upon a positive blood culture, to identify the pathogen and determine its antibiotic susceptibility pattern, but for better survival and outcome, simple and rapid diagnostics tests are required as adjuncts to the blood culture for early and effective initiation of treatment to the septicemia in neonates.

Majority (51.7\%) of CRP positive patients were found normoglycemic, $33.9 \%$ were found hypoglycemic and only $14.4 \%$ were found hyperglycemic. It was observed that $82.73 \%$ patients were cured and $17.27 \%$ were died.

C-reactive protein $\geq 6 \mathrm{mg} / \mathrm{dl}$ was considered as positive result for sepsis screen. Predictive accuracy of CRP of this study is compared with other studies. In present study, CRP positive $236(53.6 \%)$ and negative 204(46.4) were found in suspected sepsis. CRP proved to be the most efficient of all the markers of sepsis. The principal ligand to CRP is phosphocholine, which is found in lipopolysaccharide, bacterial cell walls, as well as in most biological membranes [17]. CRP is part of the acute-phase response which aims to neutralize the inflammatory agent and to promote the healing of the injured tissue [18].

Thus male babies were more affected by suspected neonatal sepsis than female babies. In the part to biological difference it is proposed to be due to genetic origin for the origin of the sex different in vulnerability to infection. The special source of vulnerability open to females by virtue of her possession of to ' $\mathrm{X}$ '-chromosomes in contrast to the single ' $\mathrm{X}$ ' of the male. Hence it is genetic locus on the ' $\mathrm{X}$ ' Chromosomes involve with synthesis of immunoglobulins responsible for the sex difference as per Thomas C. Washburn et al., [19].

Bacterial infection stimulates the hepatocytes to produce CRP: a nonspecific immune response, which is a useful clinical marker for the individual hostpathogen interaction. Since the half-life of CRP is less than 3 days, a rapid fall is seen with successful therapy. Early diagnosis of neonatal sepsis is very difficult when it is based only on clinical signs. The clinical profile is neither uniform nor specific and could mislead the health care professionals. Keeping in mind the mortality caused by neonatal sepsis, empirical treatment should not be delayed but again this can result in unnecessary and prolonged exposure to antibiotics in this early age group. Cost effective and rapid diagnostic tool is one way to tackle this issue. CRP and leukocyte count should be considered to decrease neonatal mortality rate in the country.

\section{CONCLUSION}

CRP is a simple cheap and relatively sensitive, specific with good negative predictive value in the prediction of neonatal sepsis and can be useful test in the settings with limited resources.

\section{REFERENCES}

1. Bang AT, Bang RA, Bactule SB, Reddy HM, Deshmukh MD. Effect of home-based neonatal care and management of sepsis on neonatal mortality: field trial in rural India. Lancet.1999; 354:1955-61.

2. Stoll BJ. The global impact of neonatal infection. Clin Perinatol 1997; 24:1-21. 3. Plazek MM, White LA. Early and late neonatal septicemia. Arch Dis Child. 1983;58:728-31.

3. Plazek MM, White LA. Early and late neonatal septicemia. Arch Dis Child. 1983;58:728-31.

4. Barrett BA, Hill PI. A micromethod for the erythrocyte sedimentation rate suitable for use on 
venus or capillary blood. Journal of clinical pathology. 1980 Nov;33(11):1118-20.

5. Chapagain RH, Acharya R, Shrestha N,Giri BR, Bagale BB, Kayastha M. Bacteriological Profile of Neonatal Sepsis in Neonatal Intermediate Care Unit of Central Paediatric Referral Hospital in Nepal. J Nepal Health Res Counc. 2015;13(31):205-208.

6. DoHS. Government of Nepal, Ministry of health and population. Annual report Kathmandu government of Nepal ministry of health and population department of health services, 2071/72. 2072.

7. Stoll BJ, Shane AL. Infection of the neonatal infant. In: Behrman RE, Kliegman RM, Stanton BF, III JWSG, Schor NF, Behrman RE, editors. Nelson textbook of pediatrics. 19th ed. Philadelphia: Elseveir Saunders. 2015;909-25.

8. Government of the People's Republic of Bangladesh. Students' Handbook on Integrated Management of Childhood Illness. Dhaka; 2011.

9. Aggarwal R, Sarkar N, Deorari AK, Paul VK. Sepsis in the newborn. The Indian Journal of Pediatrics. 2001 Dec 1;68(12):1143-7.

10. Bonadio WA, Hennes HA, Smith DO, Ruffing RO, Melzer-Lange MA, Lye PA, Isaacman D. Reliability of observation variables in distinguishing infectious outcome of febrile young infants. The Pediatric infectious disease journal. 1993 Feb;12(2):111-4.
11. Bekhof J, Reitsma JB, Kok JH, Van Straaten IH. Clinical signs to identify late-onset sepsis in preterm infants. Eur Journal Pediatr, 2013;172:501.

12. Weitkamp JH, Aschner JL. Diagnostic use of C-reactive protein (CRP) in assessment of neonatal sepsis. Neoreviews, 2005;6:e508-15.

13. Shah BA, Padbury JF. Neonatal sepsis: An old problem with new insights. Virulence, 2014;5:170-8.

14. Garg D, Agrawal N. Aetiology and presentation of neonatal septicaemia at tertiary care hospital of southern Rajasthan. International journal of medical science and education, 2014;1:12-20.

15. Jain NK, Jain VM, Maheshwari S. Clinical Profile of Neonatal Sepsis. Kathmandu Univ Med J, 2003;1:117-16.

16. Shrestha NJ, Subedi KU, Rai GK. Bacteriological Profile of Neonatal Sepsis: A Hospital Based Study. J Nepal Paediatr Soc, 2011;31:1-5.

17. Volanakis JE. Human C-reactive protein: expression, structure, and function. Mol Immunol, 2001;38:189-97.

18. Jaye DL, Waites KB. Clinical applications of Creactive protein in pediatrics. Pediatr Infect Dis Journal, 1997;16:735-46.

19. Washburn TC, Medearis DN. Childs, sex difference in susceptibility to infection ped. Pediatr. 1965;35(1):57-64. 